\title{
An ADHOC Based Wireless Network System
}

\author{
M.Nagalakshmi
}

\begin{abstract}
Fiasco watching is a champion the diverse maximum hard software in some distance flung uncommonly delegated frameworks as putting in established order based totally frameworks are neither feasible nor suitable in that conditions. In the direction of disaster, setbacks commonly take secures in packs in some nearby sheltered zones (as an instance, some university systems, and quick tents in a few terrific us of a regions, and so forth.). Thusly, the post catastrophe help assignments and route of benefits are regularly guard pushed. Thusly, dynamic after and engrossing sanctuary data (concerning open useful useful resource, required beneficial useful resource, reputation of setbacks and volunteers at the sheltered residence, and so on.) at a manage station is essential for great need examination from the piecewise positive points of view at every safe haven. Anyhow, in a regular fiasco circumstance, correspondence shape gets irritated snappy and the cell accessibility may be pitifully open in multiple areas of the catastrophe struck locale. As such, control correspondence of safe haven information to control station is restrained. The objective of this assignment is to have a examine using reducing aspect cellular cellular phone based keen framework for following and acclimatizing cozy houses' information and supporting the correspondence with manage station. In this splendid situation, the power of mitigation government/salvage car/police van with phones assume a easy element to make the specified ground-breaking correspondence established order.
\end{abstract}

\section{Index Terms: ADHOC, Wireless Network System}

\section{INTRODUCTION}

Some distance flung trades and frameworks organisation is one of the fastest growing regions which are up 'til now lacking appealing experienced professionals. To interface this hole, we recommend to begin investigate practices in a ways off region in our foundations wherein we can supply the understudies and researchers a location with fundamental systems/workplaces for getting fingers-on introduction and acknowledgment with the propelling advances and measures in remote correspondence and frameworks enterprise business enterprise. A number one and capability software program locale of a long way flung correspondence and flexible frameworks organisation is catastrophe management. All around, all the international locations is by using and through taking physical games to make trends for early forewarning before catastrophe and publish-failure corporation. On this manner, researchers are with the aid of and via taking powerful eagerness to cope with the traumatic conditions acted in a post-calamity scenario when the correspondence device gets resentful pretty or completely.

With a particular real objective to permit disaster to make certain and recovery wearing events after any cataclysm, fruitful correspondence the diverse special spare government, and additionally offering gadget to survivors is a crucial

Revised Manuscript Received on April 12, 2019.

Dr. M.Nagalakshmi, Marri Laxman Reddy Institute of Technology and Management, Dundigal, Hyderabad, Telangana, India critical. In a standard catastrophe circumstance, the available correspondence resource is noticeably confined and heterogeneous in nature, (as an instance, couple of WiFi towers, sat-telephones, propelled cell telephones, PDA towers). A flexible unrehearsed a long way off framework may be fashioned, at this condition with the Bluetooth interface of mobile phones passed on through help workers. The ones types of frameworks are formed mishandling the compactness of the PDA customers because the open entryway for correspondence. This all at once deployable a long way off framework can provide the quality records aggregation development that gives professionals better detectable fine of open sources and want.

PDAs remodel into an vital little bit of an man or woman's lifestyles as reducing aspect cell based totally absolutely applications like, adaptable coping with a file, territory primarily based absolutely groups, and internet diversions, and lots of others are becoming thousands of omnipresence. Ventures are proper now searching out arranged paintings with presentation in cutting facet cell growth application development in a perfect global in light of android set up.

\section{LITETATURE REVIEW}

[Braunstein 06] B. Braunstein, T. Trimble, R. Mishra, B. Manoj, L. Lenert, and R. Rao,Sanctuary course: are proposed A clever-telephone based completely secure haven manage tool for put up-ordinary disaster comfort Operations utilizing Opportunistic wireless Networks "is arranged which will assist fiasco salvage and restoration sports after any debacle, compelling correspondence many of the diverse salvage employees, simply as giving availability to survivors is an important necessity. In an average calamity scenario, the on hand correspondence asset is restricted and heterogeneous in nature, (as an instance, couple of WiFi towers, sat-telephones, cellular telephones, wi-fi towers). A transportable specifically appointed a long way flung device can be formed on this example with the Bluetooth interface of mobile telephones conveyed through help worker's. The ones styles of structures are framed abusing the portability of the PDA clients because the open door for correspondence. This brief deployable a long way flung gadget can deliver the exceptional information collecting innovation that offers experts higher perceivability of accessible property and need.

[Dilmaghani 08] R. B. Dilmaghani, and R. R. Rao, are proposedWhen a debacle or crisis happens, one of the most squeezing desires is to build up a correspondence arrange for the specialists on call at the scene. Setting up and getting to a stable correspondence framework at an emergency internet site on line is massive a great manner to have particular and consistent exchange of facts. Unhappiness within the 
shopping for and promoting of convenient and pivotal data or postponement in dispensing assets hinders early reaction endeavors, likely bringing approximately dying toll and similarly economic impact. At a fiasco internet site on line, the cutting-edge correspondence framework is probably harmed and on this way in element or certainly inaccessible; or, there may not have been previously present basis (as because of faraway zones). A correspondence framework in the putting of disaster packages have to be strong, successfully configurable, energetic, interoperable in a heterogeneous scenario with least interdependencies, and suddenly deployable effortlessly. A calamity scene is a disorganized area which requires a methodical manner to address dynamic the framework, ponder the development of facts and coordinated effort among severa controls and purviews to encourage response and recuperation endeavors. We have conveyed the a ways off artwork basis in some drills on the university grounds and within the town as a feature of the California Institute for Telecommunications and information technology (Calit2) NSF-supported RESCUE challenge (Responding to Crises and unexpected events). To evaluate set up execution and distinguish the source(s) of bottleneck, we've got got caught the machine web site site visitors. The carrying occasions gained from proving floor checks of the device depending on true conditions can be associated with future programs to enhance the gadget plan and execution.

[Hui 08] P. Hui, J. Crowcroft, and E. Yoneki, are proposedthe expanding entrance of savvy devices with systems control capacity shape novel systems. Such structures, likewise alluded as pocket exchanged structures (PSNs), are irregularly associated and communicate to a trade in outlook of sending facts in an impromptu manner. The social shape and cooperation of clients of such gadgets manipulate the exhibition of guidance conventions in PSNs. Preserving that during mind, social information is a essential size for planning sending calculations for such styles of systems. Beyond strategies relied on shape and fresh directing tables to adapt to dynamic device situations. At the downside, it's been shown that such methodologies land up being fee inadequate because of the midway trap of the transient device behavior. An all of the extra encouraging technique is capture the herbal attributes of such systems and use them in the plan of steering calculations. On this paper, we abuse social and auxiliary measurements, in particular centrality and community, using proper human portability follows. The commitments of this paper are -overlay. Inside the first place, we plan and inspect BUBBLE, a novel social-based sending calculation, that uses the formerly referred to measurements to improve conveyance execution. Second, we observationally show that BUBBLE can appreciably decorate sending execution contrasted with various currently proposed calculations which incorporates the benchmarking records-based totally definitely PROPHET calculation, and social-based completely sending SimBet calculation.

\section{BACKGROUND}

\section{A. Disaster management}

Catastrophe management is defined as 'the combination of allactivities required to build, preserve and enhance thecapabilities to put together for, reply to, get over, ormitigate in opposition to a disaster'. Those 4 sports interest onrisk control (prevention, preparedness) and crisismanagement (response and healing) comprise the disastermanagement cycle as cited in parent 1 . Theseactivities are not impartial and sequential; virtually responseand restoration stages initiate right away, whereaspopulations have one-of-a-kind prolonged-time period or quick-term recoveryoperations can pass on for days to months. Furthermore, publichealth and monetary restoration techniques can take years orbeyond that. The remaining success of response and recoveryactivities are inspired by means of way of the records amassed in the course of thepreparedness and prevention degrees.

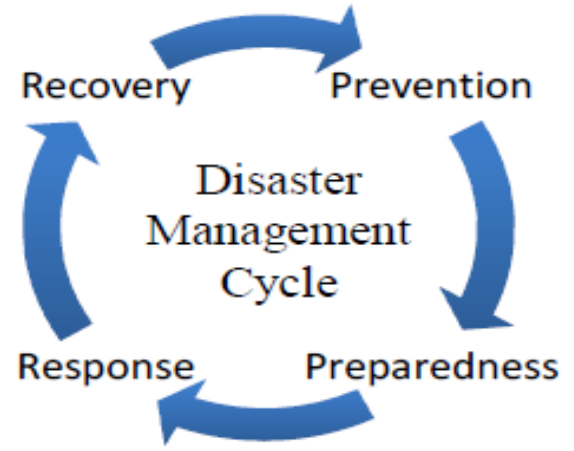

Fig.1. Phases of disaster management cycle

Fiasco the executives is a planned machine with crucial aimto reduce the adverse outcomes and impact of disasters,consequently defending humans and social framework [7].Catastrophe responsive is one of the most extraordinary degrees ofdisaster the board and opt for giving short help tomaintain existence and bolster the spirit of the affectedpopulation. That permits you to beautify fiasco responsive, it isimportant sooner or later of the arena to extend studying ofdisaster the executives. The complete above intention can also additionally befacilitated through joining extensive facts frameworks to procedure andstore non-stop voluminous disaster information with reduced time andcost for opportune simple management.

\section{B. An define of wireless Adhoc Networks}

A transportable mainly appointed device is an accumulation of flexible hubs shaping an impromptu tool with out the assist of any targeted systems. The ones structures gift every different craft of gadget basis [Das 2000, Jinyang2000] and may be appropriate for a site wherein each the framework is out of place or in which a framework is not very financially savvy. The whole lifestyles-cycle of impromptu systems may be ordered into the essential, 2nd, and the 1/three era mainly appointed tool frameworks. Present especially appointed structures frameworks are appeared because the $1 /$ three generation. The real returns to 1972. Spherical then, they have been known as PRNET (Packet Radio Networks). Related [Conti 2003, Conti 2004] 
with ALOHA (Areal locations of hazardous Atmospheres) and CSMA (service enjoy Medium get proper of entry to), techniques for medium get admission to control and a form of separation vector directing PRNET have been utilized on a initial premise to provide various structures management capacities in a struggle scenario. The second one era of in particular appointed systems rose in 1980s, at the same time as the impromptu gadget frameworks have been moreover improved and completed as a piece of the Survivable Adaptive Radio Networks software. This gave a bundle modified machine to the transportable fight region in a domain without foundation. This software examined to be useful to enhancing the radios' exhibition by way of the use of making them littler, much less high priced, and bendy to digital assaults.

\section{Programs of wireless mainly appointed Networks}

At a few aspect factor every other innovation or device develops for open use, the number one inquiry is by means of manner of what technique will it assist me or what would it no longer be able to perform for me? Right here we are able to check on this section approximately the unique blessings from programs [Xiuli 2006, Hyung 2006, Zhou 1999] of a ways flung systems within the private/Public and government division. Based at the benefits, the innovation appears to be in particular vital to the overall populace.

On this planet as of late we used to see round us distinct real uses of especially appointed a ways flung systems, however the essential subject is the automatic combat quarter. Inside the strategic condition, portable hubs can be character soldiers, established weapons, rocket/rockets launchers, trucks, helicopters, bolster motors, flying machines within the sky and even satellites at higher rises. Every awesome sort of substance has one of a kind correspondence capacities. Alongside those lines, it's miles realistic to expect that the whole tool is a heterogeneous scenario. In this circumstance, numerous types of flexible hubs will have numerous forms of movement conduct. Therefore, an adaptable, compact and loose versatility degrees are required to illustrate this $1 / 2$ of and $1 / 2$ of motion examples parent 1.Depicts using some distance off especially appointed structures decide(a) characteristic the packages area of impromptu systems and the figure (b) portrays the facts's about the harmful state of affairs like flood, seismic tremor and so forth. A part of the wireless Networks applications are as beneath:

- Tactical Networks

Navy Communications and responsibilities controls within the fight sector circumstance

- Sensor Networks

A) Collections of mounted sensor gadgets used to accumulate non-prevent statistics to robotize each potential of the framework.

B) weather tracking.

C) Earth improvement detecting wearing occasions

D) Ocean Engineering or certainly understood as Underwater Sensor Networks

- Emergency services

A) seek and salvage responsibilities and calamity healing.
B) affected man or woman document recovery

framework.

C) Catastrophic fiasco.

- Industrial surroundings

A) E-exchange and online invoice installment framework

B) get right of entry to the customer data from the fields

C) Vehicular specially appointed Networks framework.

- Instructional programs

Video conferencing framework and virtual examine hall framework

- enjoyment applications Video on hobby

- different packages

A) mobile smartphone

B) Bluetooth System

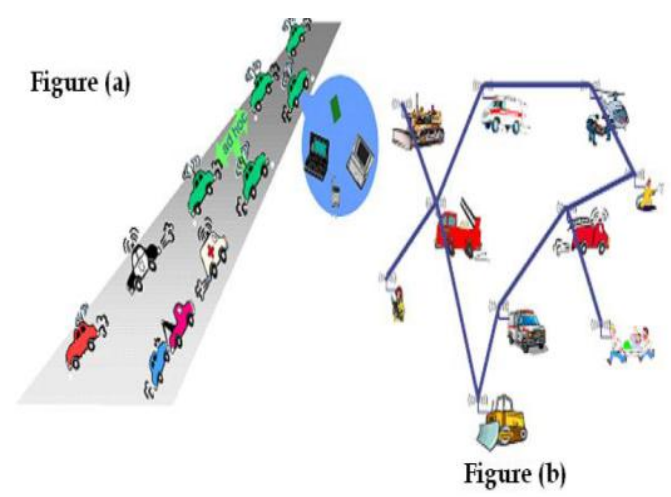

Figure 1.: Applications of Wireless Networks

\section{CHALLENGES \& ISSUES IN WIRELESS ADHOC NETWORKS}

In this innovative time, while it seems to be definitely dependable at the innovation so now actual prerequisite for the reducing part faraway device is portability the board and execution improve [Pathan 2006, Xukai 2002, Padmavati 2009, Rouba 2005] which are the primary issue. Portability the executives indicates the capacity of the system to discover a wi-fi hub, steering coming near/lively cells/information(s) paying little thoughts to its device cause of connection and hold up affiliation at the same time as the a long way flung hub wanders in distinct various systems or system's circle. There are positive studies methodological issues that should be examined to enhance the portability the executives and execution of imagined people to return again. The under composed vicinity will supply the statistics with admire to the problems seemed by the wireless specifically appointed Networks correspondences frameworks. The exam on MANET safety continues to be in its starting length. The present day proposition are normally assault located in that they first of all distinguish some protection risks and after that improve the modern-day convention or suggest any other conference to frustrate such risks. For the cause that preparations are established expressly in view of positive assault fashions, they characteristic admirably in the sight of 
assigned attacks but may fall beneath unforeseen assaults. Because of this, a progressively aspiring objective for mainly appointed device protection is to build up a multi-fence safety affiliation this is implanted into conceivably each element within the machine, coming approximately in top to backside guarantee that offers numerous strains of guard in opposition to numerous each recognized and difficult to understand protection dangers. This new plan factor of view is the element that we name versatility organized protection shape.

\subsection{Technical and studies demanding conditions}

Faraway specially appointed Networks having some specialized and studies troubles that want correction. However the reality that WANET's having this type of massive sort of blessings,[Wiechao 2003, Pathan2006, Haubaux 2004], for instance, self-reconfiguration, self mending and flexibility to pretty factor hubs attributes, as an instance, vitality effectiveness over information and information transmission and burden adjusting. The ones blessings present new problems which particularly live inside the flightiness of system topology due to the power of hubs, which, blended with the nearby communicated ability, problems in convention structure for correspondence framework, in particular appointed far off structures. To govern such form of difficulty, many checked potential methodologies have been proposed: Dynamic directing, carrier region Protocol, wireless format Protocol, and nature of-administration (QoS) based totally completely steering technique.

\subsection{Research based totally definitely annoying conditions}

Be that as it can, numerous other research areas are super together with: conventions, protection, confinement, topology control, reliability, self-alignment, self-recovering, records collection, bunch the executives, clock synchronization, query managing, sensor getting prepared [NIST 8000-186], mixture below restricted limits, and trying out and troubleshooting. A ways flung structures are an fascinating location with super capability. The effect of this territory at the arena can same the effect that the internet has had. Energizing and tough studies problems lie earlier before this movements towards becoming truth. Far flung Networks are restrained in their strength, calculation, and correspondence capacities. As unfavorable to standard machine, set up hubs are frequently despatched in available territories, displaying a threat of bodily assaults. A key to the improvement of WSN is raising the diploma of reflection for software engineers. Right now, software engineers manipulate too many low ranges insights approximately detecting and hub to hub correspondence. For instance, they regularly manipulate detecting statistics, intertwining records and shifting records. They control unique hub to hub correspondence and subtleties. Inside the event that we raise the diploma of deliberation to think about universal behavior, application usefulness and direct help for scaling troubles then profitability increments.

\section{TECHNIQUE \& RESULTS}

The proposed propelled cellular based totally correspondence device structure:

In this fragment, we will gift a quick composition diagram on the vital problems in structure up correspondence and frameworks business enterprise establishment and information organization inpost calamity situation. We study the imprisonments of top tier took after through the inducement and attention of our proposed endeavor.

Use of Bluetooth-enabled clever cellphone as data Station (storeroom of safe haven fame) to preserve revived asylum information at every safe haven specialists have currently examined the upside of using on hand far flung frameworks (additionally referred to as, sharp framework) for a couple of challenge crucial applications like, military correspondence, disaster correspondence, vehicular exchanges, and so forth. Insignificant exertion high-quality mobile phones are via and with the aid of growing with developing enrolling ability, excessive storing breaking element and various a ways flung correspondence interfaces (GSM/WiFi/Bluetooth, and so on.). In this employer, we capture the opportunity to manhandle the ones limits of a propelled mobile to make an optionally available adaptable correspondence backbone in an exasperates correspondence situation like failure.

We suggest to use a propelled cellular cellphone with blue-teeth interfaces in 3 sections at every sanctuary.

- Blue-teeth engaged PDA, set at every secure haven, will characteristic as InfoStation for that haven. All of the material statistics about an asylum is totaled at this InfoStation.

- Blue-tooth engaged propelled mobile cellular telephone, set at every haven, will characteristic as InfoStation for that covered house. All the cloth records about a secure haven is collected at this InfoStation.

- Ambulance/Police Van organized wireless can also in addition bypass about as a deliver vessel to bypass on and trade facts amongst certainly one of a kind safe homes on the identical time as shifting transversely over cozy homes.

A section of the facts that may be secured at InfoStation is recorded beneath (decide 2)

- location of a safe house

- name of the gatherings walking there (institution-Ids/codes is probably assigned previously by means of the focal workplace)

- call of the exploited humans presently last in that haven

- name of the worn out unfortunate casualties and form of ailments they're experiencing

- call of the unlucky casualties who wishes quick restorative consideration

- name of the dead humans discovered spherical there

- name of the humans of that location despite the fact that untraceable

- modern load of sustenance, drug, garb and other important products in that asylum

- facts about the accessible wellspring of savoring water that area and so forth.

- variety of cylinder wells ought to be steady direly around there and so on. 
Above statistics may be transferred to or downloaded from the InfoStation by the blue-tooth empowered PDA conveyed with the aid of the help worker's/consolation cars. The information can likewise be despatched to the remote manage station for checking with the proposed pioneering system framework.

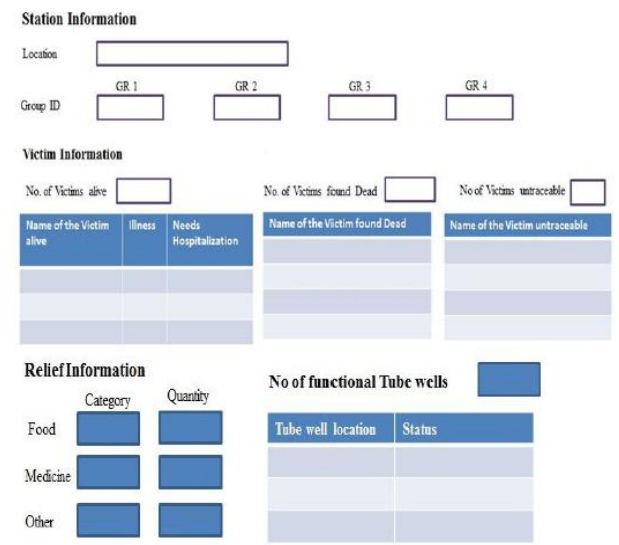

Figure 2. Station Information Chart

Exchange the message or information amongst InfoStation and alleviation expert among an asylum for altering/Storing, evaluation making use of Multimode Interface (Bluetooth + GSM/GPRS) of advanced cell phone:

The Bluetooth-empowered cellular phone will paintings right here as message perusers/pupils/bearers.

data Acquisition and information management system at Host system

A statistics-Acquisition software ought to be created to maintain jogging on the host computer at the focal office as a way to amass the statistics got from diverse havens and will display the ahead-wondering information. As and while new information about a safe residence is gotten, at that point the framework will refresh the comparing data within the host database and will display the refreshed data on the GUI.

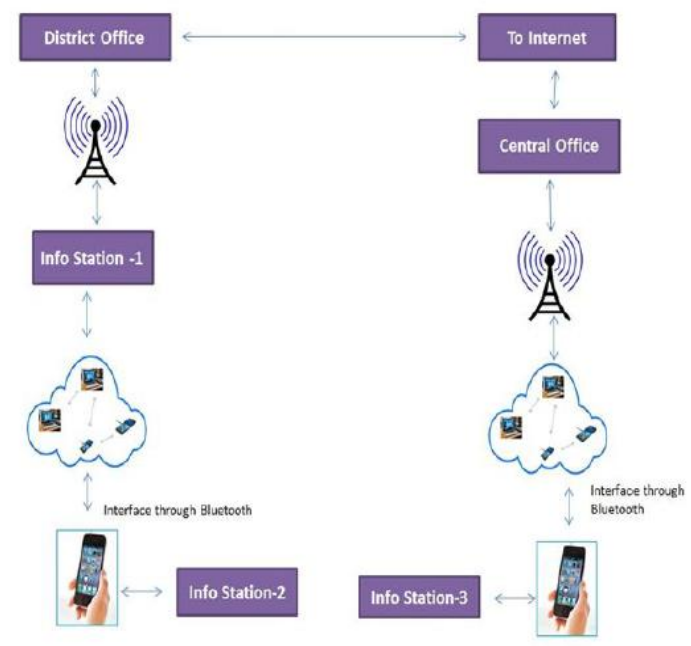

Figure 3.: Proposed Architecutre of Remote Data Acquisition system.

\section{CONCLUSION}

The catastrophe organisation the use of a ways flung incredibly delegated framework has been discussed in this paper. Using phones to make an adhoc framework can be an exceptionally convincing detail in coping with the correspondence issues in emergency conditions. Interior the stylish condition in recent times, PDAs are the most usually used devices for correspondence. In the event that there ought to be an event of an emergency state of affairs like a catastrophe, the correspondence set up is devastated at this factor an big segment of the little gadgets just like the mobile phones are saved. The difficulty is that the destruction of bendy towers makes these devices needless as correspondence isn't feasible without the supply of cell frameworks. On this form of scenario if the PDA adhoc orchestrate is surrounded, it is able to pass about as a giant correspondence form.. An vital development in the direction of this manner is develop unique packages that would make it lots less inquiring for to set up cellular cellular phone adhoc frameworks. There are various problems left for future art work, one in all the hassle is the strategies with the resource of which to help strong message transmission with considering stress and transportability.

\section{ACKNOWLEDGMENT}

This paper is submitted as part of research project "Real Time Disaster Management using ADHOC Sensor Networks" is sponsored and funded by Department of Science and Technology(DST), Science and Engineering Research Board (SERB), New Delhi. I would extend my sincere thanks to DST for Supporting this research Work

\section{REFERENCES}

1. [Airjaldi] Empowering people group through far off structures [Online]. To be had: http://drupal.Airjaldi.Com.

2. [Ben-David 10] Y. Ben-David, M. Vallentin, S. Fowler, and E. Brewer, "Jaldimac - taking the separation further," in fourth ACM Workshop on Networked structures for growing regions, pp. 1-6,2010.

3. [Braunstein 06] B. Braunstein, T. Trimble, R. Mishra, B. Manoj, L. Lenert, and R. Rao,"challenges in making use of of disseminated a long way flung artwork prepare in disaster response," in 3rdInternational ISCRAM conference, pp. 30-38, 2006.

4. [Dilmaghani 08] R. B. Dilmaghani, and R. R. Rao,"A wi-fi Mesh Infrastructure Deployment with software for Emergency scenarios," in complaints of the 5th worldwide ISCRAM convention,pp 1-eleven, 2008 .

5. [Heimerl 10] okay. Heimerl and E. Brewer, "The town base station," in fourth ACM Workshop on Networked structures for growing regions, 2010.

6. [Hossmann 11] T. Hossmann, F. Legendre, P. Carta, P Gunningberg, and C. Rohner, "Twitter in disaster mode: Opportunistic correspondence and conveyance of sensor data in crises," in ExtremeCom, 2011.

7. [Hui 08] P. Hui, J. Crowcroft, and E. Yoneki,"BUBBLE Rap: Social-primarily based definitely Forwarding in put off Tolerant Networks," in ACM MobiHoc, 2008.

8. [Hughes 08] A Hughes,LPalen, J Sutton, S Liu and S

9. Vieweg, "website online-Seeing in catastrophe: An examination of on-line Social Convergence"In lawsuits of ISCRAM,2008.

10. [Hughes 09] A Hughes and L Palen, " Twitter Adoption and Use in Mass Convergence and Emergency events," in complaints of ISCRAM,2009.

11. [Knoche 11] H. Knoche, P. SheshagiriRao, and J. Huang, "Human targeted plan for development," in global magazine of cell Human laptop interaction, vol. 3, 2011. 